Edizione cartacea:

Biblioteconomia : guida classificata / diretta da Mauro Guerrini ; condirettore Gianfranco Crupi ; a cura di Stefano Gambari ; collaborazione di Vincenzo Fugaldi ; presentazione di Luigi Crocetti. - Rist. rivista. - Milano : Editrice Bibliografica, 2008. - xl, 1143 p. : ill. — (I manuali della biblioteca ; 5). — ISBN 978-88-7075-634-0. — Prima ed.: 2007.

\title{
025.3 Functional Requirements for Bibliographic Records (FRBR)
}

\author{
Sommario \\ Origini \\ FRBR. Final Report \\ Il modello E-R \\ o Entità \\ o Attributi \\ o Relazioni \\ Funzioni utente \\ BLNBR \\ Prospettive \\ o FRAR \\ o FRSAR \\ Il dibattito \\ FRBR. Ritorno al futuro
}

\section{ORIGINI}

Alla fine degli anni Ottanta molti esperti di biblioteconomia denunciarono la necessità di rivedere criticamente i Principi di Parigi del 1961, riprendendo la riflessione sull'oggetto della catalogazione e sulla funzione della registrazione (o record). Nel corso del Seminar on Bibliographic Records tenuto dall'IFLA a Stoccolma nel 1990, i partecipanti presero atto del bisogno di ridurre i costi della catalogazione e riconobbero che la continua spinta all'uso di un livello minimo di descrizione richiedeva un'accurata revisione dei rapporti fra i singoli elementi della registrazione bibliografica e le esigenze degli utenti. L'IFLA decise di promuovere la costituzione di un gruppo di lavoro con l'obiettivo di presentare uno studio che delineasse in termini chiari e definiti le funzioni svolte dalle registrazioni rispetto ai differenti media, alle varie applicazioni e alle possibili necessità dell'utente. La prima bozza (1991) del rapporto Terms of reference for a study of the Functional Requirements for Bibliographic Records venne modificata e approvata dallo Standing Committee dell'IFLA Section on Cataloguing alla Conferenza IFLA di New Delhi del 1992, mentre il documento definitivo venne approvato alla 63a Conferenza IFLA di Copenaghen del 1997 e pubblicato a metà 1998 con il titolo Functional Requirements for Bibliographic Records. Final report (FRBR), <http://www.ifla. org/VII/s13/frbr/frbr.pdf>. Il Rapporto è in fase di ulteriore elaborazione da parte del FRBR Review Group dell'IFLA. 


\section{FRBF. FINAL REPORT}

FRBR s'inserisce in quella great tradition (tradizione di qualità) che inizia con Antonio Panizzi e prosegue con Charles Coffin Jewett, Charles Ammi Cutter, Shiyali Ramamrita Ranganathan, Andrew D. Osborn, Seymour Lubetzky, Eva Verona, Ákos Domanovszky, caratterizzata dal comune interesse nei confronti degli objects (nel duplice senso di oggetti e obiettivi) della catalogazione; rispetto a quella tradizione, FRBR introduce tuttavia un elemento di novità, in quanto pone attenzione alla registrazione bibliografica piuttosto che al catalogo nel suo insieme. FRBR tenta di elaborare un modello concettuale che: a) permetta d'identificare i requisiti minimi della descrizione che interessano all'utente nella consultazione di una registrazione in un catalogo o in una bibliografia; b) definisca le finalità della registrazione e le modalità della sua struttura, in relazione ai tipi di media e alle molteplici necessità degli utenti.

FRBR ha due obiettivi principali:

1. fornire una cornice strutturata e chiaramente definita per mettere in relazione i dati selezionati nelle registrazioni catalografiche con le esigenze degli utenti;

2. raccomandare un livello base di funzionalità per le registrazioni create dalle agenzie bibliografiche nazionali.

Il Rapporto è costituito da una parte introduttiva, a cui segue il nucleo principale dello studio, articolato in due sezioni: presentazione del modello entità-relazioni (E-R); raccomandazioni per un livello minimo delle registrazioni nelle bibliografie nazionali (BLNBR, Basic Level National Bibliographic Record, Requisiti di base per le registrazioni bibliografiche nazionali).

\section{IL MODELLO E-R}

Per individuare gli oggetti d'interesse di chi consulta le registrazioni catalografiche - dunque per stabilire l'oggetto della catalogazione - e il modo e le finalità con cui le registrazioni vengono utilizzate, FRBR cerca di rappresentare l'universo bibliografico tramite un modello E-R (entità-relazioni), un tipo di modello concettuale elaborato nell'ambito del database design da Peter Pin-Shan Chen (The Entity-Relationship model: towards a unified view of data, "ACM transactions on database systems", vol. 1, no. 1 (1976)) e Richard Perkinson (Data analysis: the key to data base design, Wellesley, Mass., QED Information Sciences, 1984). Le diverse entità sono poste in relazione tra loro e possono essere descritte per mezzo di elementi di dati (o anche proprietà, caratteristiche), detti attributi.

\section{Entità}

Un'entità è definita come elemento che presenta interesse in un dato contesto. Le entità individuate in FRBR sono gli oggetti primari dell'interesse degli utenti dei dati bibliografici e dei prodotti del lavoro intellettuale o artistico, riconducibili agli elementi tradizionali, descrittivi o d'indicizzazione, della registrazione catalografica. FRBR divide le entità in tre gruppi:

Gruppo 1 i prodotti dell'attività artistica o intellettuale: opera, espressione, manifestazione, item; 
Gruppo 2 i responsabili del contenuto intellettuale o artistico, della produzione fisica e della diffusione o della tutela di tali prodotti: persona ed ente;

Gruppo 3 le entità che fungono da soggetti del lavoro intellettuale o artistico, ovvero da soggetti delle opere: concetto, oggetto, evento, luogo, più le entità del Gruppo 1 e 2 quando costituiscono il soggetto di un'opera; per esempio, un'opera su un'altra opera o su una persona.

I Gruppi 2 e 3 esistono solo per effetto delle loro relazioni con il primo e sono in corso di precisazione da parte di specifici sottogruppi nati da FRBR: FRANAR, Functional Requirements and Numbering of Authority Records, denominato FRAR dal 2004, e FRSAR, Functional Requirements for Subject Authority Records, costituito nel 2005. Il Gruppo 1 comprende:

Opera: la creazione intellettuale o artistica originale, priva di materializzazione; potremmo pensare a essa - afferma FRBR - come alle idee nella mente di una persona;

Espressione: la realizzazione intellettuale o artistica di un'opera nella forma alfanumerica, musicale, coreografica, sonora, visiva, oggettuale, in movimento, ecc. o qualsiasi combinazione di queste forme; le espressioni sono le modalità con cui sono state espresse le idee o il contenuto di un'opera; espressione è la realizzazione di un'opera tramite un codice (p.e., il testo modificato, aggiornato, ampliato, emendato, tradotto di un'opera);

Manifestazione: l'oggettivazione dell'espressione di un'opera; l'espressione che viene diffusa e pubblicata, rappresenta tutti gli oggetti fisici che mostrano le stesse caratteristiche di contenuto intellettuale e forma fisica (p.e., l'Eneide di Virgilio pubblicata a Milano da Garzanti nel 1984);

Item: l'esemplare, la singola copia di una manifestazione, l'unità elementare di un insieme di copie identiche (p.e., una copia dell'Eneide di Virgilio edita a Milano da Garzanti nel 1984 [l'edizione italiana di FRBR - Requisiti funzionali per record bibliografici, Roma, ICCU, 2000 - traduce l'inglese item con documento].

Le entità definite come opera ed espressione riflettono un contenuto intellettuale o artistico; le entità definite come manifestazione e come item riflettono la forma fisica. La manifestazione ha infatti una valenza astratta (come l'opera e l'espressione), ma rappresenta entità fisiche, concrete, costituite dall'item (nel caso di un dipinto) o da tutti gli item (nel caso di un libro prodotto in mille copie) che la realizzano. L'opera esiste soltanto nella comunanza di contenuto fra le sue varie espressioni. Patrick Le Boeuf, responsabile del FRBR Review Group dal 2002 al 2005, presenta un esempio assai chiarificatore per comprendere le entità. Egli parte correttamente dall'elemento concreto - l'item - per arrivare all'elemento più astratto - l'opera - in un processo di astrazione progressiva: "Quando diciamo "libro" abbiamo in mente un oggetto fisico distinto formato dalla carta e da una rilegatura e che a volte può servire per tenere aperta una porta o da rincalzo sotto la gamba di un tavolo. FRBR lo chiama item. Quando diciamo 'libro' forse vogliamo dire anche 'pubblicazione', come nel caso in cui ci rechiamo in libreria per chiedere un libro identificato da un ISBN; di solito per noi la copia specifica non ha importanza purché faccia parte della classe generale di opere che cerchiamo e che non ci siano pagine mancanti. FRBR lo chiama manifestazione. Quando diciamo 'libro' come nella domanda "chi ha tradotto questo libro?" forse abbiamo in mente un testo specifico in una data lingua o una traduzione. FRBR 
lo chiama espressione. Quando diciamo 'libro' come nella domanda "chi ha scritto questo libro?" forse intendiamo anche un livello più alto di astrazione, il contenuto concettuale (intellettuale o artistico) che è alla base di tutte le versioni linguistiche, la storia di fondo narrata nel libro, le idee nella mente di una persona per un libro. FRBR lo chiama opera" (Patrick Le Boeuf, Brave new FRBR world, relazione presentata all'IME ICC di Francoforte sul Meno nel 2003, <http://www.ddb.de/ standardisierung/pdf/papers_leboeuf.pdf $>$ ). Le entità del Gruppo 1 sono tra loro legate da un rapporto ad albero: un'espressione è la realizzazione di una e soltanto di un'opera e può essere materializzata in una o più manifestazioni; così come una manifestazione può oggettivare una o più espressioni. Una manifestazione, a sua volta, può essere esemplificata da uno o più item, ma un item può esemplificare soltanto una manifestazione.

\section{Attributi}

A ciascuna entità definita dal modello è associato un insieme di caratteristiche o attributi (detti metadati in altri contesti). Gli attributi di un'entità sono le proprietà tramite cui gli utenti formulano richieste e interpretano i risultati della ricerca bibliografica; hanno un corrispettivo nei campi delle maschere di ricerca degli opac. La scelta degli attributi, contestuale all'ambito di applicazione di FRBR, è il risultato di un'analisi dei dati tipici di una registrazione, condotta principalmente su ISBD e GARE, Guidelines for Authority and Reference Entries, un documento sulla redazione di registrazioni d'autorità edito dall'IFLA nel 1984 e, in seconda edizione nel 2001, con il nome di GARR, Guidenlines for Authority and Reference Records. Gli attributi sono divisi in due grandi categorie: inerenti all'entità o esterni a essa. La prima comprende le caratteristiche fisiche, gli aspetti formali che connotano e distinguono un'entità (p.e., la formulazione della notizia così come compare sul frontespizio, sulla copertina o sulla custodia di un libro). La seconda comprende gli identificativi assegnati all'entità (p.e., il numero del catalogo tematico di una composizione musicale). L'attributo "nome della persona", per l'entità persona, può assumere valori diversi a seconda delle occorrenze (p.e., pseudonimi, abbreviazioni, traduzioni, traslitterazioni).

\section{Relazioni}

«Nel contesto del modello le relazioni servono come veicolo per rappresentare il legame tra un'entità e un'altra e quindi come mezzi per aiutare l'utente a "navigare" nell'universo che è rappresentato da una bibliografia, da un catalogo o da un database bibliografico" (FRBR, 5.1). Le relazioni consentono all'utente di individuare, di localizzare, mediante una ricerca:

- $\quad$ tutte le opere di un autore;

- tutti i libri pubblicati in una serie (collana);

- tutti gli atti dei congressi di una società;

- $\quad$ tutti i materiali, indipendentemente dalla loro forma, che trattino un soggetto.

Le relazioni collegano dunque le entità tramite i loro attributi e possono essere registrate implicitamente - aggiungendo alla registrazione un punto d'accesso relativo all'entità correlata - o esplicitamente - tramite una nota descrittiva che indichi la natura specifica della relazione, p.e., "Tradotto dal testo inglese 
dell'edizione del 1891". Anche le relazioni sono di due tipi: inerenti e di contenuto. Le relazioni inerenti sono quelle intrinseche al modello: un'opera "si realizza" in una o più espressioni, un'espressione "si materializza" in una o più manifestazioni, e una manifestazione "rappresenta" uno o più item; un'opera "è creata da" una persona o un ente, un'espressione "è realizzata" da una persona o un ente, una manifestazione "è prodotta da" una persona o un ente e un item "è posseduto da" una persona o un ente; un'opera "ha per soggetto" un concetto, un oggetto, un evento o un luogo. Le relazioni di contenuto operano a livello di opere ed espressioni e vengono poi trasmesse transitivamente alle relative espressioni e manifestazioni e all'item. Tra di esse vi sono quelle che riguardano il grado di prossimità di contenuto tra entità diverse: abbiamo allora relazioni

equivalenti, quando siamo nell'ambito di una stessa espressione;

derivative, quando la differenza concettuale tra due entità è tale da richiedere una nuova espressione;

descrittive, quando si ha a che fare con opere correlate ma diverse, fino a formare intere famiglie di opere.

Altre relazioni di contenuto sono quelle di continuità, supplementarietà e complementarietà (p.e., una nota di un supplemento può riferirsi a un'espressione precedente dell'opera).

\section{FUNZIONI UTENTE}

Nel 1876 Charles A. Cutter identificò diversi obiettivi del catalogo. FRBR li riprende in una nuova prospettiva, per rispondere al meglio alle possibili esigenze e attività di un utente nei confronti di una registrazione bibliografica; FRBR individua quattro funzioni utente:

Trovare l'entità che corrisponde ai criteri stabiliti dalla ricerca su un soggetto o dalla ricerca di una manifestazione identificata con un titolo particolare;

Identificare un'entità, cioè confermare che la manifestazione descritta in una registrazione corrisponde alla manifestazione desiderata o distinguere fra due testi o fra due registrazioni che hanno lo stesso titolo;

Selezionare un'entità che corrisponde alle proprie necessità, per esempio selezionare un testo nella propria lingua o scegliere una versione di un programma per computer compatibile con l'hardware e con il sistema operativo a disposizione;

Ottenere o acquisire accesso all'entità descritta: compilare l'ordine di acquisto di un libro, inoltrare la richiesta per il prestito di un CD-ROM, accedere a un documento elettronico presente in un computer remoto.

Una quinta funzione di carattere più generale è stata poi individuata in Navigare (ovvero Correlare): l'utente deve infatti poter navigare nell'universo delle entità rappresentato da un catalogo o da una bibliografia, per poter reperire altre opere correlate a un'opera per mezzo di generalizzazioni, associazioni, aggregazioni e attributi correlati per equivalenza, associazione e rapporto gerarchico (cfr. Elaine Svenonius, The intellectual foundation of information organization, Cambridge, Mass., The MIT Press, 2000; ma a sottolineare l'importanza del browsing fu già a suo tempo A.C. Foskett in The subject approach to information, $2^{\text {nd }}$ ed. revised 
and enlarged, London, Bingley, 1971, p. 25); la funzione verrebbe soddisfatta dal ruolo che il modello assegna alle relazioni.

\section{BLNBR}

Il modello E-R facilita indubbiamente la comprensione delle attuali convenzioni per la registrazione catalografica delle risorse documentarie, ma FRBR va oltre: raccomanda un livello base di funzionalità per le registrazioni create dalle agenzie bibliografiche nazionali (per l'Italia la BNI). Incrociando attributi e relazioni da un lato, e funzioni utente dall'altro, tramite precise tabelle, FRBR individua i dati di base da includere nelle registrazioni delle agenzie bibliografiche nazionali (BLNBR, Requisiti di base per i record bibliografici nazionali); i requisiti sono divisi in due grandi categorie: elementi organizzativi (punti di accesso) ed elementi descrittivi. FRBR ricorda, inoltre, la necessità di prevedere livelli diversi di completezza delle registrazioni, ovvero eventuali ulteriori attributi e relazioni, secondo il contesto a cui sono destinate (p.e., una citazione bibliografica rispetto a una registrazione in un catalogo di biblioteca). FRBR lascia volutamente aperte alcune questioni, dall'analisi dell'indicizzazione delle registrazioni d'autorità (authority record), curata dal gruppo FRANAR, a quella dell'indicizzazione per soggetto, di cui si occupa dal 2005 il gruppo FRSAR, Functional Requirements for Subject Authority Records. La pubblicazione di FRBR ha suscitato un dibattito molto vivace nella comunità bibliotecaria, tale da richiedere nel 2002 la costituzione di un FRBR Review Group, che coordina il dibattito internazionale, redige una bibliografia sul tema (<http://www.ifla.org/VII/s13/wgfrbr/bibliography.pdf>) e cura una mailing list di discussione (<http://www.ifla.org/VII/s13/wgfrbr/archive/FRBR_Listserv_Archive.pdf>).

\section{Prospettive}

\section{FRAR}

Nello stesso anno della pubblicazione di FRBR, due importanti incontri, il seminario su The function of bibliographic control in the global information infrastructure (Vilnius, Lituania, 17-19 giugno 1998) e l'International Conference on National Bibliographic Services (Copenhagen, 27-29 novembre 1998) segnarono un rinnovato interesse per l'authority control, tanto che l'IFLA costitui un nuovo gruppo di lavoro, FRANAR, Functional Requirements And Numbering of Authority Records, finalizzato all'estensione del modello di FRBR alle registrazioni d'autorità (punti di accesso alle registrazioni catalografiche). Il gruppo stabilì tre obiettivi principali:

1. definire i requisiti funzionali delle registrazioni d'autorità;

2. studiare la fattibilità di un ISADN, International Standard Authority Data Number;

3. fungere da collegamento tra l'IFLA e altri gruppi con interessi connessi (p.e., CERL, <Indecs>, LEAF). 
Nel 2001 il gruppo decide di accantonare il progetto di un ISADN, e nel 2004 di cambiare la sua denominazione in FRAR, Functional Requirements of Authority Records; tra il 2003 e il 2005 pubblica due draft, il secondo dei quali ha per titolo Functional Requirements for Authority Records. A conceptual model. FRAR ha un duplice scopo:

1. facilitare la comprensione di come gli authority files operino attualmente;

2. chiarire i concetti fondamentali dell'authority work per migliorarne la prassi.

FRAR introduce nell'ambito delle entità di suo interesse - il Gruppo 2 - le nuove entità famiglia: nome, identificativo, punto di accesso, regole e agenzia, ciascuna delle quali prevede specifiche relazioni inerenti. Le stesse funzioni utente cambiano per meglio adattarsi al diverso contesto: oltre a trovare e identificare sono presenti le nuove funzioni contestualizzare (contestualizzare le entità; chiarire le relazioni fra persone, enti e opere e chiarire le relazioni fra queste e i nomi con cui sono conosciute) e giustificare (documentare i motivi delle decisioni del catalogatore relativamente alle forme del nome scelte come punti di accesso), che sostituiscono selezionare e ottenere di FRBR.

\section{FRSAR}

Il gruppo, Functional Requirements for Subject Authority Records (FRSAR), è stato costituito nel 2005 e si occupa delle registrazioni di soggetto. Esso ha stabilito tre obiettivi principali:

1. costruire un modello concettuale delle entità del Gruppo 3: concetto, oggetto, evento, luogo;

2. fornire una cornice di riferimento per correlare i dati delle registrazioni di soggetto alle esigenze degli utenti;

3. valutare il potenziale del modello per l'uso e la condivisione internazionale dei dati delle registrazioni di soggetto in ambito bibliotecario ed extrabibliotecario (p.e., archivi, musei).

FRSAR introduce numerose novità: aggiunge le entità di stato e di tempo (tempo è legato a evento); l'oggetto di percezione o percepito (in ingl. percept), nel senso di ciò che può essere percepito dai sensi. Si tratta di una proposta, in linea peraltro con quanto va affermandosi nelle RDA, Resource Description and Access (le nuove AACR, Anglo-American Cataloguing Rules), curate da Tom Delsey, insieme editore nel nuovo codice catalografico e del gruppo di lavoro su FRSAR; l'oggetto di percezione sostituisce ciò che era semplicemente denominato oggetto. 


\section{IL DIBATTITO}

La pubblicazione di FRBR. Final report ha suscitato un vivace dibattito nella comunità bibliotecaria e delle professioni dell'informazione in genere. La principale questione è stata l'interpretazione della natura e delle finalità del modello: analitico-descrittive o normativo-prescrittive? $\mathrm{E}$ cioè FRBR un'analisi concettuale dei record così come sono, oppure intende prospettare una nuova struttura dei record? A questo dibattito sono collegate le riflessioni sul tipo di modello scelto (E-R) e sulle entità individuate da FRBR, ritenute da alcuni troppe o troppo poche, da altri di difficile resa in termini pratici. Riguardo alla prima questione, è il testo stesso a generare ambiguità; FRBR 1.3 prospetta infatti un modello che serva da "spunto per una serie di studi a seguire che possano essere di particolare interesse per coloro che sono coinvolti nella elaborazione di codici di catalogazione e di sistemi di supporto alla creazione, gestione ed uso di dati bibliografici" (p. 5 e 14 dell'ed. italiana). Difficile poi stabilire se FRBR 7 (BNLBR, Requisiti di base per i record bibliografici nazionali) vada interpretato come semplice scomposizione concettuale di quanto attualmente troviamo già nei record bibliografici nazionali o come modello per nuove registrazioni. L'ipotesi normativo-prescrittiva, che ha a sua volta generato la percezione di FRBR come inizio di una rivoluzione nel mondo della catalografia, fu nel 2000 fatta propria dalla Section on Cataloguing dell'IFLA, la quale propose di avviare una revisione a tutto campo delle ISBD per conformarle a FRBR. Da qui un impegnato dibattito internazionale sulle differenti visioni di FRBR e della sua implementazione. Il confronto si è incentrato soprattutto sulla struttura del record - rimarrà composita o sarà scomposta in diverse registrazioni, ciascuna relativa a un'entità componente? (il catalogo perderebbe l'attuale struttura lineare per acquisirne una reticolare?), e sulle entità rispecchiate dalle registrazioni (riguarderanno la manifestazione, come sembra lecito aspettarsi in un ambiente di catalogazione partecipata, oppure l'item, come ha suggerito Patrick Le Boeuf all'IME ICC di Francoforte nel 2003?). 
Il processo di catalogazione inizia infatti dalla descrizione dell'item, a partire dal quale è possibile risalire per astrazione alle essenze (le entità di FRBR) di cui esso si compone. La questione ha inoltre un risvolto problematico per quanto riguarda le risorse elettroniche ( $\mathrm{su}$ cui mancano esempi in FRBR), dato che per esse è difficile (e forse anche poco utile) stabilire cosa sia l'opera, cosa l'espressione e cosa la manifestazione, così come quale sia la differenza (se c'è) tra manifestazione e item; in particolare, il carattere mutevole delle pagine web rende difficile una loro trattazione secondo il modello di FRBR. A ciò è inevitabilmente collegata la diatriba sulle entità stesse (e più precisamente quelle del Gruppo 1): c'è chi ritiene troppo sintetica l'entità espressione, racchiudendo questa diversi livelli di prossimità concettuale tra loro non sufficientemente distinti ed essendo indispensabile distinguere almeno l'espressione capostipite dalle altre; dall'altro c'è chi reputa di dubbia praticità e validità la differenza tra opera ed espressione, per l'impossibilità di fissare un limite preciso nel discriminare l'opera dall'espressione o per il carattere estremamente astratto dell'opera; c'è inoltre chi giudica necessaria l'introduzione dell'entità super-opera o di un super-record (Martha Yee, Elaine Svenonius e Rahmatollah Fattahi, autore di Relevance of cataloguing principles to the online environment. An historical and analytical study, School of Information, Library and Archive Studies, University of New South Wales, Sydney, 1997, <http://web.um.ac.ir/ fattahi/thesis 1.htm>), necessari per poter raggruppare opere distinte ma facenti parte di un'unica "famiglia" (p.e., adattamenti di un'opera, periodici che cambiano nome, continuazioni) e opere aggregate (tipiche dell'ambito musicale); similmente c'è chi (Le Boeuf, ma la questione era già a suo tempo sostenuta da Diego Maltese) segnala l'importanza del riconoscimento della natura intenzionale delle pubblicazioni che raccolgono più opere, viste non più come semplici contenitori materiali di opere tra loro diverse, bensì come insiemi concettuali rispondenti a una precisa volontà dell'editore o del curatore (super-manifestazioni? In realtà si tratta di una diversa concezione della manifestazione che la sottrarrebbe alla dicotomia intellettuale/materiale che divide in due le entità del Gruppo 1). Altri elogiano FRBR per aver fatto chiarezza sul tormentato concetto di edizione (che corrisponde a espressione se riguarda differenze testuali, e a manifestazione se riguarda diverse emissioni di un testo), evidenziando nel contempo l'impossibilità di tradurre tale acquisizione concettuale nella struttura delle ISBD, le cui otto aree sono trasversali alle entità di FRBR, a meno di non voler rivedere totalmente le ISBD, come ha ipotizzato lo stesso Michael Gorman, loro ideatore. Altra questione dibattuta è quella del tipo di modello scelto per FRBR: c'è infatti chi afferma che un modello O-O (Object Oriented), di più moderna concezione, è più appropriato del modello E-R. Una posizione più distaccata è infine quella di Alfredo Serrai (Critica dei Functional Requirements for Bibliographic Records, "Bibliotheca", 2002, n. 2, p. 207-215), che rivendica la paternità delle entità del Gruppo 1, criticando FRBR per il suo operativismo ingenuo e per aver modellato l'analisi concettuale sulle prassi utenziali invece di fondarsi sull'esigenza di un'adeguata rappresentazione bibliografica del documento.

\section{FRBR. RITORNO AL FUTURO}

Risultato più evidente di quest'ampio dibattito è stata la divaricazione delle diverse percezioni di FRBR a seconda del campo di applicazione. 
In ambito di normative catalografiche (e in seno all'IFLA) ha col tempo prevalso l'interpretazione che ridimensiona FRBR a un quadro concettuale di analisi dei record per valutarne il livello di soddisfacimento rispetto alle esigenze dell'utente e per fissare una terminologia condivisa sugli oggetti descritti dalle registrazioni: il programma della Cataloguing Section IFLA di allineare ISBD a FRBR si è così limitato a una lieve riduzione degli elementi descrittivi considerati obbligatori (p.e., il complemento del titolo, non prescritto da BLBNR come obbligatorio) e a una mappatura fra FRBR-ISBD e FRBR-MARC. Il documento ricorda che ISBD e FRBR occupano piani differenti e si limita a mostrare che la stragrande maggioranza degli elementi dei record (gli attributi di FRBR) riguarda il solo livello della manifestazione. A questo proposito Barbara Tillett osserva: "OCLC ha compiuto alcune ricerche per stabilire quanti dei propri record comportano relazioni con opere, espressioni e manifestazioni. È emerso che meno del $20 \%$ di tutti i record rappresenta opere con più di una manifestazione. Guindi probabilmente arriveremo alla conclusione che il singolo record bibliografico a livello di manifestazione è tutto ciò che ci occorre per la grande maggioranza dei record che creiamo. Tuttavia avremo bisogno di fornire informazioni aggiuntive a livello di opera/ espressione in caso di manifestazioni multiple per poter raggruppare i record nelle visualizzazioni del nostro catalogo" (Barbara Tillett, FRBR e VIAF. Esempi delle attuali iniziative internazionali sulla catalogazione, in Studi e testimonianze offerte a Luigi Crocetti, Milano, Editrice Bibliografica, 2004, p. 686). Circoscritto alla terminologia risulta poi l'impatto di FRBR sulle nuove regole di catalogazione angloamericane (RDA, Resources Description and Access, con il chiaro intento di presentarsi come codice internazionale), che prevedono (draft del 2005) di adottare, sotto l'etichetta di Functional objectives, le funzioniutente Identificare e Selezionare e, accanto al termine più generico di risorsa, le entità del Gruppo 1 di FRBR.

Per quanto riguarda le Regole italiane di catalogazione per autori, la Commissione RICA ha deciso di non accogliere la terminologia di FRBR, la cui analisi concettuale delle entità verrà tuttavia utilizzata per l'impostazione della struttura del codice, che procederebbe così dal concreto (la descrizione dell'esemplare che si ha in mano) all'astratto (caratteristiche relative ai livelli sovraordinati). La Commissione RICA ha infatti "esaminato la possibilità di un'applicazione ridotta e progressiva del modello FRBR, che preveda la creazione iniziale di una sorta di registrazione temporanea che comprenda i dati basilari e sia successivamente suscettibile di arricchimenti, quando ne sussistano le condizioni" (Isa de Pinedo, Alberto Petrucciani, Un approccio all'applicazione del modello FRBR alle regole di catalogazione italiane: problemi e possibili soluzioni, "Bollettino AIB», vol. 42, n. 3 (lug-set. 2002), p. 267-278). 
Ben oltre il ruolo analitico è invece andato FRBR negli altri ambiti: il nuovo millennio ha rivelato la profonda influenza di FRBR sia sui principi internazionali, sia a livello di opac e applicativi, sia ancora sui modelli esterni all'ambito strettamente bibliografico. Lo Statement of International Cataloguing Principles prodotto dall'IME ICC, International Meeting of Experts on an International Cataloguing Code, di Francoforte sul Meno (2003), dichiara di volere aggiornare i Principi di Parigi alla luce dei concetti e della terminologia di FRBR (e FRAR). Il campo più battuto per l'applicazione di FRBR resta tuttavia quello dello sviluppo dei database, come fin dall'inizio prospettò Delsey, e degli applicativi in genere. I primi esempi di applicazione di FRBR per la visualizzazione dei record sono stati: AustLit, Australian Literature Electronic Gateway (un database, non basato su ISBD, di record relativi alla letteratura australiana, che parte da un'opera e ne mostra le relazioni con espressioni e manifestazioni, e usa anche l'entità superopera); la Library of Congress, con l'FRBR Display Tool (<http://www.loc.gov/marc/marc-functional-analysis/tool.html>), che visualizza i record in MARC 21, distribuendo le informazioni sui diversi livelli di opera, espressione e manifestazione. FRBR ha suscitato interesse anche nelle comunità professionali degli archivi e dei musei (cfr. FRBR/CIDOC CRM Harmonization Group).

\section{Testo}

Functional requirements for bibliographic records : final report / IFLA Study Group on the Functional Requirements for Bibliographic Records ; approved by the Standing Committee of the IFLA Section on Cataloguing. - München : Saur, 1998. - <http://www.ifla.org/VII/s13/frbr/frbr.pdf>.

Requisiti funzionali per record bibliografici : rapporto conclusivo / IFLA Study Group on the Functional Requirements for Bibliographic Records ; approvato dallo Standing Committee della IFLA Section on Cataloguing ; edizione italiana a cura dell'Istituto centrale per il catalogo unico delle biblioteche italiane e per le informazioni bibliografiche. - Roma : ICCU, 2000.

Per approfondire

Introduzione a FRBR : Functional Requirements for Bibliographic Records = Requisiti funzionali per record bibliografici / Carlo Ghilli, Mauro Guerrini. Milano : Editrice Bibliografica, c2001.

Seminario FRBR : Functional Requirements for Bibliographic Records = Requisiti funzionali per record bibliografici, Firenze, 27-28 gennaio 2000 : atti / a cura di Mauro Guerrini = FRBR Seminar : Functional Requirements for Bibliographic Records $=$ Requisiti funzionali per record bibliografici, Florence, 27th-28th January 2000 : proceedings / edited by Mauro Guerrini. - Roma : Associazione italiana biblioteche, 2000. 